\title{
SOME COMPARTMENTALIZED SECURE TASK ASSIGNMENT MODELS FOR DISTRIBUTED SYSTEMS
}

\author{
Daniel C. Lee, Member, IEEE
}

\begin{abstract}
This paper formulates abstract problems of assigning subtasks to agents (processors) in a distributed system with a goal that they can perform its global task efficiently. The paper models the distributed system with a graph that describes the communication capabilities of the constituting agents. This graph is referred to as the "organizational graph." In addition, the desired task-performing activity is modeled with another graph describing the required communications. Then, a few variants of the task assignment problem are formulated with potentially conflicting objectives (or constraints) of load balancing and communication costs. For some of these variants this paper provides efficient algorithms that solve the assignment problem. Some problems are proven NP-complete, and some others are left open.
\end{abstract}

Index terms- Task assignment, distributed systems, load balancing, complexity, information security

D. C. Lee is with the School of Engineering Science at Simon Fraser University, 8888 University Drive, Burnaby, BC V5A1S6, Canada.

E-mail: dchlee@sfu.ca

Some part of this work was presented in Proc. Fourth International Conference on Knowledge-Based Intelligent Engineering Systems \& Allied Technologies, University of Brighton, UK, Aug. 2000. 


\section{INTRODUCTION}

A distributed computing system designed to perform a particular global task accomplishes its objectives by partitioning that task into subtasks and assigning these to its agents (processors). Generically, some of these subtasks interact; that is, they cannot be carried out by the corresponding agents in isolation. This introduces the need for communication between certain pairs of agents. This paper focuses on such communication aspects of a distributed system. In particular, we describe the system's organization by specifying "who talks to whom" -that is, by means of an undirected graph $G_{O}=\left(V_{O}, A_{O}\right)$. The set of nodes $V_{O}=\left\{a_{1}, a_{2}, \cdots, a_{n}\right\}$ represents the set of agents, and the presence of an arc $\left(a_{i}, a_{j}\right) \in A_{O}$ signifies that agents $a_{i}$ and $a_{j}$ can communicate with each other. In this paper, we will always assume that $\left(a_{i}, a_{i}\right) \in A_{O}$ for all $a_{i}$ $\in V_{O}$, which simply expresses the fact that any agent can communicate with itself. We will refer to graph $G_{O}$ as the distributed system's organizational graph. $G_{O}$ specifies the communication capabilities available to the system. Note that $\left(a_{i}, a_{k}\right) \notin A_{O}$ indicates that agents $a_{i}$ and $a_{k}$

cannot communicate, even if $\left(a_{i}, a_{j}\right) \in A_{O}$ and $\left(a_{j}, a_{k}\right) \in A_{O}$ for some $a_{j}$. Two agents' inability to communicate with each other may model the distributed system's security constraint. In some groups, in order to prevent the leakage of secret information, the information is compartmentalized and kept separately by different agents that are prevented from communicating with each other. Such compartmentalization is common in the intelligence community as a protective measure against counterintelligence. Alternatively, such an inability to communicate may model the case in which maintaining reliable communication between two agents is prohibitively expensive.

Certain tasks might require communication among all agents of the distributed system, in which case the most suitable system organization would correspond to a complete graph. On the other hand, there are numerous situations in which the task to be executed has a special structure, in which case fewer communication links suffice. Thus, the task assignment in the distributed system is closely related to the organizational structure. For the case of fixed organizational structure (that is, the task assigner cannot control which agent can communicate with which), the subtasks must be assigned in such a way that the organizational structure can accommodate the communication requirements. In this case, the major performance measure of the assignment 
may be the balance of loads among agents. For the case in which the task assigner also has authority over the organizational structure, the task assignment is not constrained by the fixed organizational structure. However, each assignment requires a specific structure of inter-agent communications (i.e., organizational structure). The cost of retaining the communication structure may be an additional performance criterion in this case.

To clearly define our problem, we need to mathematically represent the communication requirements of the task to be executed. This can be done in terms of another undirected graph, $G_{T}=\left(V_{T}, A_{T}\right)$, called the task graph. The nodes of $G_{T}$ correspond to subtasks while the presence of an $\operatorname{arc}(i, j) \in A_{T}$ signifies that subtasks $i$ and $j$ are interdependent. Each subtask $i \in V_{T}$ is to be assigned to an agent in $V_{O}$, the agent primarily responsible for that task. We denote by $\sigma_{i}$ the agent to which subtask $i$ is assigned. In our model, the interdependence between two subtasks $i$ and $j$ is handled by assigning to a particular agent in $V_{O}$ the responsibility of keeping track of this interdependence. (For practical illustration, the interdependence necessitates communication between two processes handling the two compartmentalized subtasks, and some agent should handle the responsibility of supervising that communication between the two processes.) We denote by $\sigma_{i j}$ the agent to which this responsibility is assigned. (For example, agent $\sigma_{i j}$ oversees cooperative activities between $\sigma_{i}$ and $\sigma_{j}$ for security purposes.) It is then natural to require that $\sigma_{i j}$ should be able to communicate with both $\sigma_{i}$ and $\sigma_{j}$. In this paper we assume that $G_{T}$ is a connected graph. (If $G_{T}$ is not connected, then it can be regarded as a collection of maximal connected subgraphs $G_{T}^{1}, G_{T}^{2}, \ldots, G_{T}^{v}$-that is, each of $G_{T}^{1}, G_{T}^{2}, \ldots$, and $G_{T}^{v}$ is a connected graph and no path exists from a node in $G_{T}^{i}$ to a node in $G_{T}^{j}$ if $i \neq j$. Each subgraph can be considered an independent project task and can be regarded as a separate task.

Formally, we have the following definition.

Definition 1: Given a task graph $G_{T}$, a valid organizational structure is defined as a graph $G_{O}$, together with a mapping $\sigma: V_{T} \cup A_{T} \rightarrow V_{O}$ with properties: $\left(\sigma_{i j}, \sigma_{i}\right) \in A_{O}$ and $\left(\sigma_{i j}, \sigma_{j}\right) \in A_{O}$ for every $(i, j) \in A_{T}$.

(We will mostly use the notation $\sigma_{i j}$ and $\sigma_{i}$ instead of the more standard functional notations $\sigma(i)$ or $\sigma(i, j)$. Also, recall that in accordance with our definition of $G_{O},\left(a_{i}, a_{i}\right) \in A_{O}$ for all $a_{i} \in V_{O}$. The task assignment problems to be considered will be of the following form: given the task graph $G_{T}$, find a valid organizational structure (the mapping $\sigma$ and graph $G_{O}$ per Definition 1) so 
as to optimize a given performance measure, subject to some additional constraints that remain to be specified. The following are some additional constraints:

- We can impose a constraint on the cardinality of $V_{O}$ - that is, on the number of available agents.

- We could assume that the graph $G_{O}$ is given, which would correspond to the case in which we are dealing with a pre-existing system organization. In this case, all that remains to be done is to design the mapping $\sigma$ in some desirable way. An implicit assumption here is that all agents of the pre-existing organization are equally capable and versatile, so that any subtask can be assigned to any agent.

- Going one step further, we could assume that the graph $G_{O}$ is given and that the agent $\sigma_{i}$, which is in charge of subtask $i$, is also pre-specified for each task $i$. In this case, we only have to choose which agent will be responsible for the handling of each subtask interaction. That is, we only need to choose the values of $\sigma_{i j}$, for every $(i, j) \in A_{T}$. Such a problem would correspond to a situation in which each subtask is of a specific nature, intimately linked to a particular agent that is the only agent capable of handling it. On the other hand, the implicit assumptions are that the handling of the interactions between subtasks $i$ and $j$ does not involve any particular expertise and that it can be handled by any agent, as long as the necessary communication links are in place.

Next, we have to specify some relevant performance criteria. Our first criterion pertains to load balancing. The agents of any distributed system have limited resources, and there is a limit to the number of their responsibilities. We assume that handling the interaction between each pair of subtasks imposes a unit processing load. We denote by $p(i)$ for each $i \in V_{T}$ the load of executing subtask $i$. Formally, we define the load $\ell_{k}$ of agent $a_{k} \in V_{O}$ to be:

$$
\ell_{k} \equiv \sum_{\left\{i \in V_{T} \mid \sigma_{i}=a_{k}\right\}} p(i)+\left|\left\{(i, j) \in A_{T} \mid \sigma_{i j}=a_{k}\right\}\right| .
$$

This is the agent $a_{k}$ 's burden of executing subtasks plus the interactions for which this agent is responsible. In this paper, we will assume that $p(i)$ is an integer for each $i \in V_{T}$. (This assumption of integer $p(i)$ and that of the unit load for the interaction between each pair are crucial to the arguments used in the following sections. Problems with more relaxed assumptions 
are left for future research.) The maximum load $L$ is defined by $L=\max \left\{\ell_{k} \mid a_{k} \in V_{O}\right\}$. $L$ is a performance criterion to be considered and smaller $L$ is favored.

Another performance criterion relates to the amount of communication resources employed by the distributed system. This is a natural measure, given that communication is often a constrained resource. In fact, we will be considering two alternative ways of measuring communication resources, as follows.

- Given a system organization $G_{O}$, let $C 1$ be the number of $\operatorname{arcs}\left(a_{i}, a_{j}\right) \in A_{O}$ for which $a_{i} \neq a_{j}$. Thus, $C 1$ measures the number of communication links that have to be in place when setting up the system.

- In an alternative method of measuring communication, we can measure the total amount of communication cost in the system. We model the fact that interactions between different pairs may need different intensities of communication. Also, the required amount of communication for subtask interactions may depend upon the task assignment. By way of illustration, for every $(i, j) \in A_{T}$, agent $\sigma_{i j}$ has to exchange messages with both agents $\sigma_{i}$ and $\sigma_{j}$ if $\sigma_{i j}$ coincides with neither. In this case, communication cost will be incurred in both links. However, if $\sigma_{i j}$ coincides with $\sigma_{i}$, then we should not "charge" for communication between $\sigma_{i j}$ and $\sigma_{i}$. Furthermore, we can imagine a system in which different links have different communication costs. Thus, we represent the communication cost for the interaction $(i, j) \in A_{T}$ between subtasks by function value $\mu\left((i, j), \sigma_{i}, \sigma_{j}, \sigma_{i j}\right)$. The total communication cost for all pairs of (distinct) agents, to be denoted by $C 2$, can be defined as $C 2=\sum_{(i, j) \in A_{T}} \mu\left((i, j), \sigma_{i}, \sigma_{j}, \sigma_{i j}\right)$. For illustration, let us consider a special case of equal communication intensity for all pairs of subtasks and equal communication cost for all links. We can model this case by defining $\mu\left((i, j), \sigma_{i}, \sigma_{j}, \sigma_{i j}\right)=2$ if $\sigma_{i} \neq \sigma_{j}$ and $\sigma_{i j} \notin\left\{\sigma_{i}, \sigma_{j}\right\}$, $\mu\left((i, j), \sigma_{i}, \sigma_{j}, \sigma_{i j}\right)=1$ if $\sigma_{i} \neq \sigma_{j}$ and $\sigma_{i j} \in\left\{\sigma_{i}, \sigma_{j}\right\}, \mu\left((i, j), \sigma_{i}, \sigma_{j}, \sigma_{i j}\right)=1$ if $\sigma_{i}=\sigma_{j} \neq \sigma_{i j}$, and $\mu\left((i, j), \sigma_{i}, \sigma_{j}, \sigma_{i j}\right)=0$ if $\sigma_{i}=\sigma_{j}=\sigma_{i j}$. Let us specialize further to the case that $\sigma_{i} \neq \sigma_{j}$ as long as $i \neq j$. That is, no two subtasks are assigned to the same agent. Then $C 2$ can be regarded as $2\left|A_{T}\right|$ minus the number of elements $(i, j)$ of $A_{T}$ for which $\sigma_{i j} \in\left\{\sigma_{\mathrm{i}}, \sigma_{j}\right\}$ - that is, 
one unit of communication cost for $(i, j)$ because one link is used if $\sigma_{i j} \in\left\{\sigma_{\mathrm{i}}, \sigma_{j}\right\}$ and two units of communication cost for $(i, j)$ because two links are used if $\sigma_{i j} \notin\left\{\sigma_{i}, \sigma_{j}\right\}$.

It should be clear that the objectives of load balancing and low communication requirements compete with each other. For example, in the special case of equal communication intensity for all pairs of subtasks and equal communication cost for all links, communication requirements are lowest if all subtasks are assigned to a single agent, which results in a very unbalanced load. In our problem formulations, we will often deal with this trade-off by attempting to optimize one of the performance measures while constraining the other. For example, we might wish to minimize $C 1$ subject to a constraint that $L$ be bounded above by some given $L_{u p}$.

The results presented by this paper are organized as follows. In sections 3,4 , and 5 , we assume that each agent can be assigned at most one subtask-i.e., a feasible mapping $\sigma$ is constrained to have property $\sigma_{i} \neq \sigma_{j}$ if $i \neq j$. As one example of a physical meaning of this constraint, an organization might prevent an individual agent from having excessive information or authority for the purpose of security (i.e., adequate compartmentalization of information and power). Each of sections 3, 4, and 5 then considers the task assignment problem under different assumptions about "how much" of $G_{O}$ and of mapping $\sigma$ are to be predetermined. For each choice of assumptions, we consider a few different problems, depending upon the particular choice of performance measure ( $L, C 1$ or $C 2$ ). Section 6 relaxes the assumption that each agent can be assigned at most one subtask, and discusses the task assignment problems.

\section{MOTIVATION AND RELATED WORKS}

The mapping, $\sigma: V_{T} \cup A_{T} \rightarrow V_{O}$, which is part of a valid organizational structure that this paper is seeking, is reminiscent of the well defined graph embedding problem [Röm96, Mon95,Diek93]. The main difference is that the graph embedding problem would seek a mapping $f: V_{T} \rightarrow V_{O}$ [Röm96]. In the present paper, we place a relatively high emphasis on information security among the purposes of distributed processing. (Compartmentalization of information is a very common practice for information security in an organization - especially an intelligence organization.) As mentioned in section 1, the present paper views the responsibility 
of supervising communication between two subtasks as a computational burden, which is separate from the communication cost. Indeed, when compartmentalization is employed as a method of information security, the information exchange between parties must be carefully guarded. The content of exchanged information should be examined to make sure that the content really needs to be exchanged. Also, the confidentiality and integrity of the exchanged information should be protected. The present paper regards the responsibility of supervising the communication, which is represented by an edge in $A_{T}$, between a pair of subtasks as an activity separate from that of performing a subtask, which is represented by a node in $V_{T}$. The present paper intends to model the task assignment of a distributed organization with high emphasis on information security.

In fact, an idea of modeling organizational behavior by parallel and distributed computation was presented earlier [Lee87]. Section 2.1 will show how the problem of finding mapping, $\sigma$ : $V_{T} \cup A_{T} \rightarrow V_{O}$, originated historically - namely, from the study of organizational behavior modeled by distributed optimization and the study of decomposing the cost function [BerTsi89,Lee87]. Section 2.2 mentions previously studied problems similar to the problem introduced in Section 1.

\subsection{Task Assignment in Distributed Organization Modeled by Decomposition of Cost Function}

We will now describe, in some detail, an example that historically motivated the mapping problem introduced in Section 1. (However, it should be noted that the mapping problem introduced in Section 1 has more a general framework than this sample problem.) In this example, the behavior of an organization is modeled by a distributed optimization algorithm [Lee87]. Consider an organization comprising agents whose objective is to come up with an $n$-dimensional decision vector $x=\left(x_{1}, \ldots, x_{n}\right)$. Each component is decided by only one agent of the organization; i.e., only one agent has the authority and responsibility over each component of the vector. Let $\sigma_{i}$ denote the agent of the organization that will be responsible for the decision $x_{i}$. We assume that the performance of a decision vector $x$ is judged according to a cost function $J$ : $R^{n} \rightarrow R$ and that the organization's aim is to choose a decision vector $x$ that minimizes $J$. Let us further assume that the organization strives toward this objective by mimicking a gradient 
algorithm. That is, a preliminary decision vector $x$ is chosen, which is then updated by making a correction along a direction of cost improvement, as in the gradient algorithm $x:=x-\gamma \nabla J(x)$.

Let us now assume that the cost function $J$ has the structure

$J(x)=\sum_{i=1}^{n} J^{i}\left(x_{i}\right)+\sum_{(i, j) \in A_{T}} J^{i j}\left(x_{i}, x_{j}\right)$

(e.g., a quadratic cost function). Here, $J^{i}$ captures the immediate cost to agent $\sigma_{i}$ due to its own decision, whereas $J^{i j}$ reflects the interactive effect of the decisions of agents $\sigma_{i}$ and $\sigma_{j}$ on the cost. The set $A_{T}$ indicates the set of all pairs of interacting agents. We assume that for every pair of interacting agents $\sigma_{i}, \sigma_{j}$, with $(i, j) \in A_{T}$, there is some agent, denoted by $\sigma_{i j}$, that will have the responsibility of measuring and suitably communicating the effects of these interactions. We assume that the cost function $J^{i}$ is known only to agent $\sigma_{i}$ for each $i$, and that $J^{i j}$ is known only to agent $\sigma_{i j}$ for each $(i, j) \in A_{T}$. (This models the organization whose agents do not know the global objective, possibly for reasons of protecting the secrecy of the organization.) In such a case, the organizational behavior can be modeled by an asynchronous or synchronous version of a distributed gradient algorithm [Tsi84]. Note that agent $\sigma_{i}$, in order to perform its variable update for the gradient algorithm

$$
x_{i}:=x_{i}-\gamma \frac{\partial J}{\partial x_{i}}(x)=x_{i}-\gamma \frac{\partial J^{i}}{\partial x_{i}}\left(x_{i}\right)-\gamma \sum_{(i, j) \in A_{T}} \frac{\partial J^{i j}}{\partial x_{i}}\left(x_{i}, x_{j}\right)
$$

needs the value of $\frac{\partial J^{i j}}{\partial x_{i}}\left(x_{i}, x_{j}\right)$ for all $j$ such that $(i, j) \in A_{T}$. Also, agent $\sigma_{i j}$ needs values of $x_{i}$ and $x_{j}$. Clearly, the communication requirements of this algorithm are that $\sigma_{i j}$ should be able to communicate with agents $\sigma_{i}$ and $\sigma_{j}$, in conformance with our general model. Note that $C 1$ measures the number of pairs of agents that need to communicate with each other. On the other hand, $C 2$ can represent the communication overhead for exchanging partial derivatives and variables that would have to be communicated between agents; both are meaningful measures of communication. Furthermore, according to our general definition, the load $\ell_{i}$ of agent $\sigma_{i}$ can reflect the computational burden of updating its variable $x_{i}$ and computing the partial derivatives that have to be evaluated by that agent during a typical iteration. 


\subsection{Module Allocation, Mapping, Graph embedding, and Scheduling Problems}

Numerous papers have been written on task matching and scheduling. (For examples, see [Braun98, ShHK95, CasKu88, Grid99, ArmHK98, AlPrRa99, MaSie98, Wang97, Fre96, BlDr96, BerW96, BhGM95, IvOzFo95, LePS95, SohnR95, BatA194, YangGe94, Weber93, PengS93, SihLee93, AngerHC90, ShWP90]). The precise optimal solutions of most scheduling problems are intractable [GareyJ79], so many papers discuss heuristic algorithms. The formulation of problems being discussed in the present paper does not explicitly consider the temporal aspect of task performance in their formulation. In other words, minimizing execution time is not explicitly a formal performance objective of our task allocation (assignment), although achieving the performance criteria defined in the present paper will strongly tend to reduce the execution time. Thus our problems, formulated as mapping between graph components, may address applications in which the distributed system performs an ongoing task (in contrast to a finite amount of computation). Or, in the case of finite computation, we can macroscopically consider load balancing as an effort to reduce execution time.

The design problems that we have formulated are reminiscent of the mapping problem in [Bok87], which is to map the vertices of the "problem graph" to the nodes of a graph modeling a parallel processor or array. However, the objective and constraint of the task assignment problem in the present paper are different. The mapping problem discussed in the present paper is also similar to a general class of problems referred to as task assignment problems or module allocation (MA) problems [Stone77, Stone78, ChuHLE80, Bok81, Gus83, Towsley86, Sinclair87, Lo88, Fer89]. The "communication graph" in the module allocation problem and the task graph $G_{T}$ in the present paper have different meaning, as our task assignment problem assigns edges of $G_{T}$ to agents (processors) as well as the vertices. Moreover, the module allocation (MA) problem minimizes the sum of the execution cost and communication cost, while the task assignment problem in the present paper addresses conflicting objectives of communication cost and load balancing.

Although independently developed, reference [Efe82] is similar to the problems presented in the present paper in that the load imposed on each process is considered as a constraint in minimizing the communication cost resulting from the module allocation. Since the processors are assumed to be homogeneous in the module allocation problem discussed in [Efe82], the constraint on the load forces the load to be balanced to a certain extent. The 
problems introduced in the present paper have different task structures, however. Also, the assumptions on the structure of the processor network are much looser than [Efe82], because [Efe82] assumes a fully interconnected network of processors.

It is worthwhile to differentiate between the task assignment problem in the present paper and the widely studied graph embedding problem [Röm96, Mon95, Diek93]. The graph embedding problem would be to find a mapping from $V_{T}$ to $V_{O}$ while considering load, dilation, and congestion as performance measures. A major differentiator of the task assignment problem in the present paper is that it seeks a mapping from $\boldsymbol{V}_{\boldsymbol{T}} \cup \boldsymbol{A}_{\boldsymbol{T}}$ to $V_{O}$. In addition, the task assignment problem has the constraint that the resulting organizational structure should be valid, as specified in Definition 1.

In the problem discussed in section 5 (the case in which the only constraint imposed on organizational graph $G_{O}$ is the number of agents, $\left|V_{O}\right|$ ), the system designer has the flexibility of designing the topology of the agents, $G_{O}=\left(V_{O}, A_{O}\right)$. Thus, the task assignment problem contains an element of graph topology design similar to a communication network's topological design problem [BerGal92]. However, there are significant differences. First, the communication requirement in the task assignment problem is determined by the designer's choice of mapping $\sigma$, whereas the amounts of data traffic between origin-destination pairs are given in the communication network topology design problem. Second, the constraint of valid organizational structure (Definition 1) greatly limits the routing of information between each origin-destination pair - namely, $G_{O}=\left(V_{O}, A_{O}\right)$ must have the property that $\left(\sigma_{i j}, \sigma_{i}\right) \in A_{O}$ and $\left(\sigma_{i j}, \sigma_{j}\right) \in A_{O}$ for every $(i, j) \in A_{T}$

\section{FIXED DISTRIBUTED SYSTEM ORGANIZATION AND EXPERTISE}

Let there be given a task graph $G_{T}$. In this section, we consider the distributed system design problem under the following assumptions:

1) the organizational graph $G_{O}=\left(V_{O}, A_{O}\right)$ is also given.

2) $\left|V_{T}\right| \leq\left|V_{O}\right|$.

3) $\sigma_{i}=a_{i}$ for all $i$ (the assignment of each subtask is given). 
(An exemplary physical meaning of this is that subtask $i$ in $V_{T}$ requires expertise that only agent $a_{i}$ has. Or, from an example of information security, only agent $a_{i}$ has access to information required to handle subtask $i$.) Thus, the task assignment problem under these assumptions is to choose the value of $\sigma_{i j}$ for every $(i, j) \in A_{T}$. First, we note the possibility that there does not exist a feasible task assignment- that is, there may not exist a mapping $\sigma$ that results in a "valid organizational structure" as defined in section I. It is easy to determine whether a feasible mapping $\sigma$ exists. In particular, we only need to check whether for every $(i, j) \in A_{T}$ there exists some agent $a_{k}$ for which $\left(a_{i}, a_{k}\right) \in A_{O}$ and $\left(a_{j}, a_{k}\right) \in A_{O}$. (Note that $a_{k}$ here can be $a_{i}$ or $a_{j}$ because $\left(a_{l}, a_{l}\right) \in A_{O}, \forall a_{l} \in V_{O}$ in our definition of $G_{O}=\left(V_{O}, A_{O}\right)$.)

\subsection{Minimizing the maximum load $L$}

The first problem we consider is the following. We wish to find a valid task assignment which minimizes the maximum load $L$, subject to the constraints mentioned in the introduction to this section.

The above defined problem can be solved in polynomial time by solving a sequence of linear network flow problems. We start by considering the following related question: given a value $L_{u p}$, does there exist a valid task assignment, satisfying all of our constraints and such that $L \leq L_{u p}$ ? This question can be answered by solving a network flow problem. The following algorithm produces the answer.

\section{Algorithm 3.1 - Phase 1}

- For each element $(i, j)$ of $A_{T}$, create a node $m_{i j}$, and for each element $a_{i}$ of $V_{O}$, create a node $d_{i}$.

- For each element $(i, j)$ of $A_{T}$ and for each node $a_{k} \in V_{O}$, if $\left(a_{i}, a_{k}\right) \in A_{O}$ and $\left(a_{j}, a_{k}\right) \in A_{O}$, then create an edge from $m_{i j}$ to $d_{k}$.

- Create the source node $s$ and make an edge from $s$ to each $m_{i j}$ with capacity limit 1.

- Create the sink node $t$

- For each agent $a_{i}$ that is assigned a subtask (i.e., $i \in V_{T}$ ), make an edge from node $d_{i}$ to $t$ with capacity limit $L_{u p}-p(i)$.

- For each agent $a_{l}$ that is not assigned with a subtask $\left(l \notin V_{T}\right)$, make an edge from node $d_{l}$ to $t$ with capacity limit $L_{u p}$. 
(A flow graph constructed by Algorithm 3.1 - Phase 1 is illustrated in Fig. 1.) Phase 2 solves the max-flow problem associated with this flow graph.

\section{Algorithm 3.1 - Phase 2}

- Run an algorithm that solves, under the constraint of integer flows, the max-flow problem constructed in Phase 1.

- If the maximal flow is $\left|A_{T}\right|$, there is a valid task assignment. Otherwise, there is not.

There are algorithms that efficiently (in polynomial time) find the maximal flow from $s$ to $t$ with the property that the flow through each link has an integer value (e.g., [PaSt82], [BerTsi97], [Ber98], [BaJS05]). We denote by variable $x_{i j, k}$ the flow through the edge from $m_{i j}$ to $d_{k}$. Then, $x_{i j, k}=1$ signifies $\sigma_{i j}=a_{k}$, and $x_{i j, k}=0$ signifies that the interaction between subtasks $i$ and $j$ is not handled by agent $a_{k}$. The flow graph imposes constraint $\sum_{k=1}^{n} x_{i j, k}=1$ for the case of max-flow $=$ $\left|A_{T}\right|$, reflecting the fact that each interacting pair $(i, j)$ in $A_{T}$ must be assigned to some agent $a_{k}$ Furthermore, since $\sigma_{i j}$ must be able to communicate to $a_{i}$ and $a_{j}$, we construct the flow graph in the following way: if either $\left(a_{i}, a_{k}\right) \notin A_{O}$ or $\left(a_{j}, a_{k}\right) \notin A_{O}$, then there is no edge from $m_{i j}$ to $d_{k}$. Through the capacity limits of the edges to $t$, we impose the constraint, $p(k)+\sum_{(i, j) \in A_{T}} x_{i j, k} \leq L_{u p}$ for each agent $a_{i}$ in $V_{O}$.

In order to find the optimal value of $L$, we could solve the above network flow problem for all values of $L_{u p}$ from $\max \left\{p(k) \mid a_{k} \in V_{O}\right\}$ to $\max \left\{p(k) \mid a_{k} \in V_{O}\right\}+\left|A_{T}\right|$, and this would still be a polynomial-time algorithm for the original problem. In fact a faster algorithm is obtained if we perform binary search for the optimal value of $L$; in particular, it would suffice to solve $O\left(\log \left|A_{T}\right|\right)$ network flow problems. 


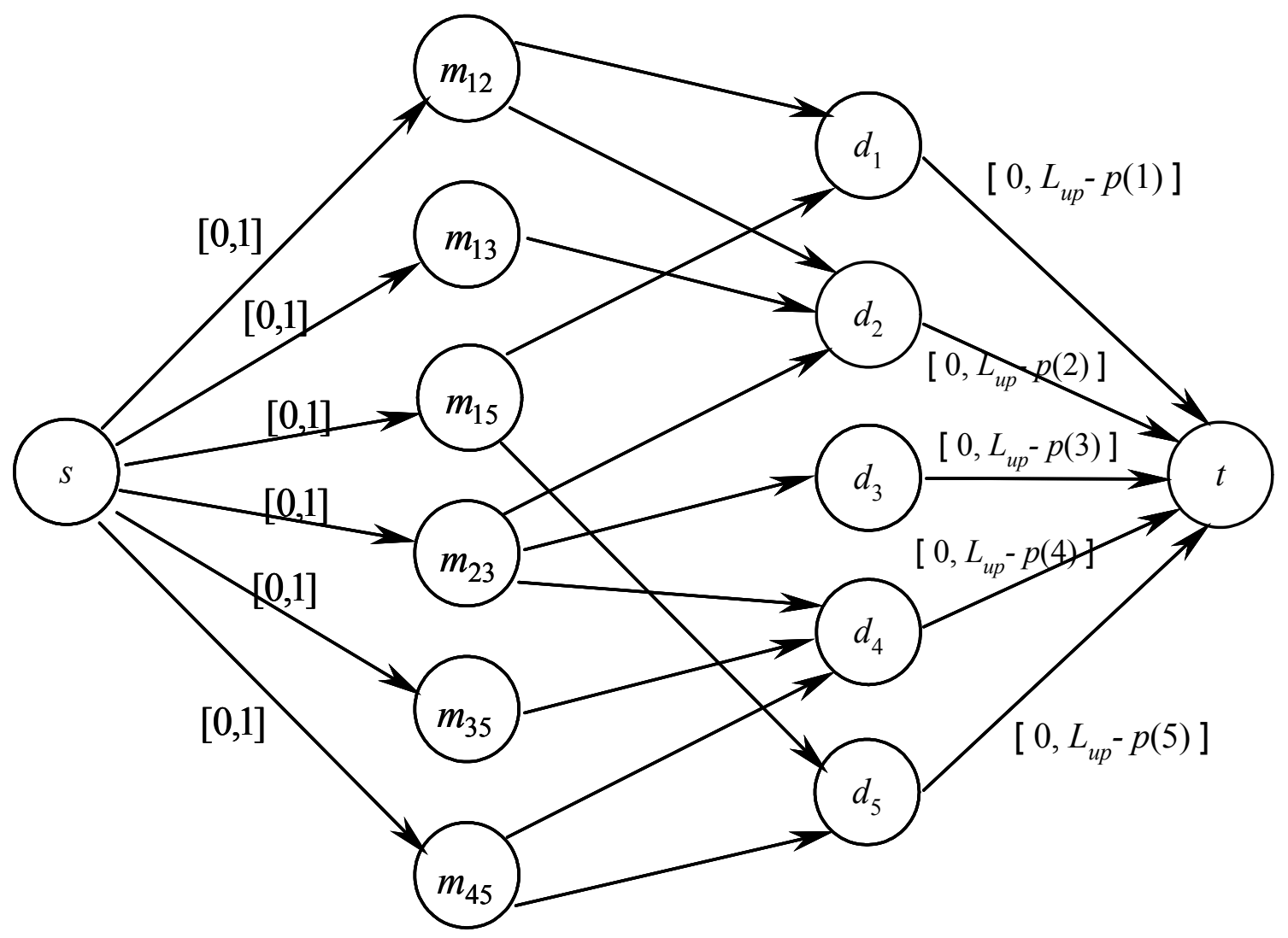

Network flow graph
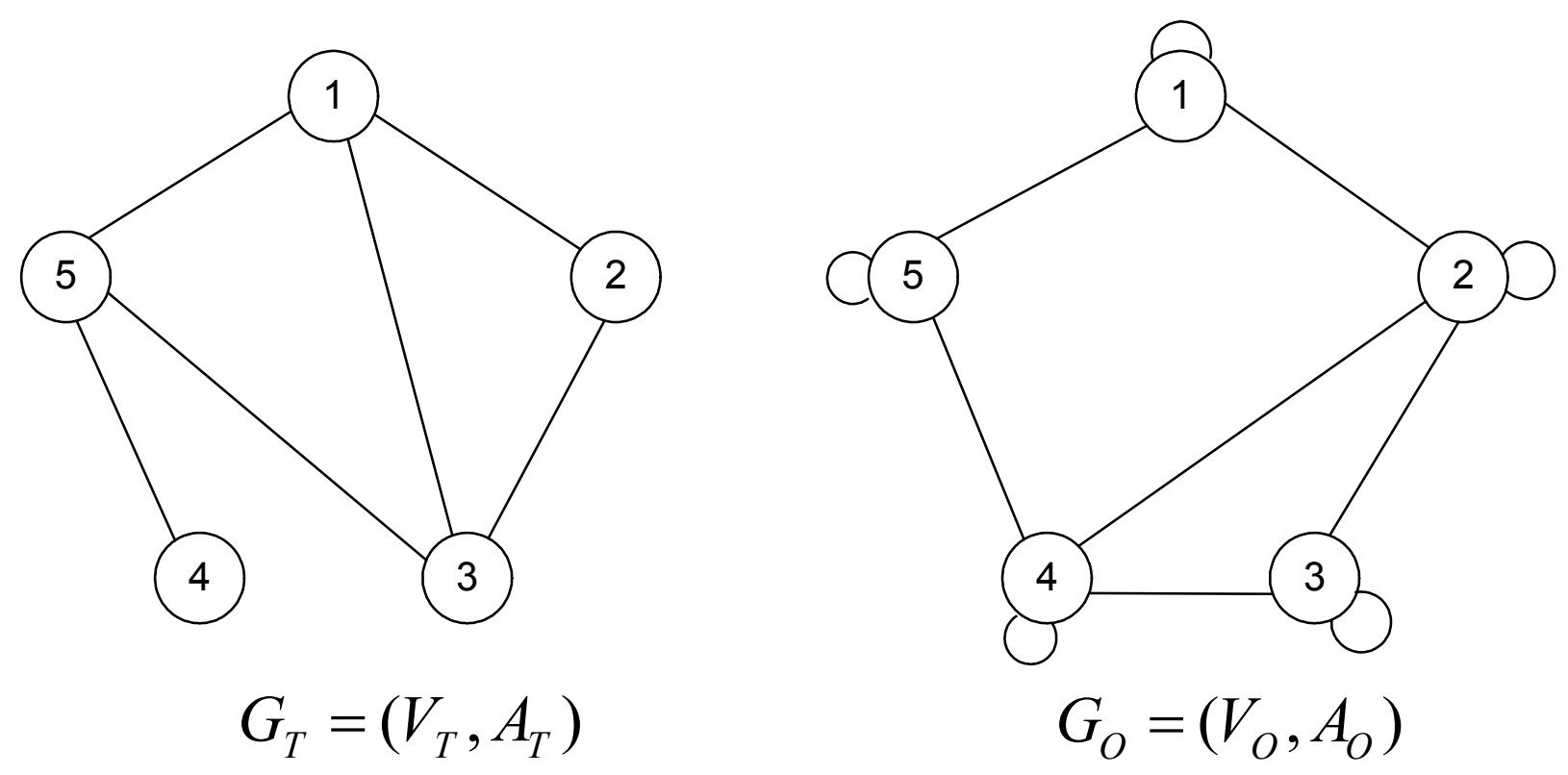

Fig. 1. Network flow graph construction 


\subsection{Minimizing a communication measure}

The problem of minimizing the number $C 1$ of arcs is vacuous because $G_{0}$ is assumed to be given and therefore $C 1$ is predetermined. The problem of minimizing $C 2$ is also very simple, as we now discuss. In the case of fixed distributed system organization, we have $C 2=\sum_{(i, j) \in A_{\tau}} \mu\left((i, j), a_{i}, a_{j}, \sigma_{i j}\right)$. Therefore, in order to minimize $C 2$, clearly we should choose assignment for each $(i, j) \in A_{T}$ :

$$
\begin{array}{r}
\sigma_{i j}=\arg \min _{\left\{a_{k} \in V_{O} \mid\left(a_{i}, a_{k}\right) \in A_{O},\left(a_{j}, a_{k}\right) \in A_{O}\right\}} \\
\mu\left((i, j), a_{i}, a_{j}, a_{k}\right)
\end{array}
$$

\subsubsection{Minimizing a communication measure under load constraints}

A more interesting problem addresses the constraint on the load imposed on the agents. We consider the problem of minimizing $C 2$ subject to an upper bound $L_{u p}$ on the maximum load $L$. This problem again can be formulated as the min-cost flow problem [PaSt82] through the following procedure:

\section{Algorithm 3.2}

- Run Phase 1 of Algorithm 3.1.

- Set the required flow to be $\left|A_{T}\right|$.

- For each combination of $m_{i j}$ and $d_{k}$ that are connected by an edge, set the cost of the edge to be $\mu\left((i, j), a_{i}, a_{j}, a_{k}\right)$.

- Set the costs of other edges to be each 0 .

Flow from $m_{i j}$ to $d_{k}, x_{i j, k}$, cannot exceed 1 from the construction of the network flow graph. Also, if we constrain $x_{i j, k}$ within $\{0,1\}$, clearly the min-cost flow of the network is the minimal $C 2$ under the load constraint $L_{u p}$. Application of the Primal-Dual algorithms in [PaSt82, Chapter 7] shows that we can achieve the minimal cost of the network constructed in Algorithm 3.2 and that the same minimal cost can be achieved by a flow vector that has an integer flow value along each edge. In order to obtain such a flow vector in the above network flow graph, we can apply polynomial-time algorithms in [PaSt82, Chapter 7]. 


\section{FIXED DISTRIBUTED SYSTEM ORGANIZATION OF VERSATILE AGENTS}

Let there be given a task graph $G_{T}$. In this section we again assume that the graph $G_{O}$ is given. However, in contrast to the preceding section, we do not impose the requirement that a particular subtask must be assigned to a particular agent. In this section, instead of constraint $\sigma_{i}$ $=a_{i}, \forall i$, which was imposed in section 3, we impose the milder requirement that each agent is assigned at most one subtask. An exemplary physical meaning of this constraint is that an organization prevents an individual agent from having excessive information or authority for the purpose of security (i.e., adequate compartmentalization of information and power). With this constraint, we can trivially conclude that there is no feasible mapping $\sigma \operatorname{if}\left|V_{T}\right|>\left|V_{O}\right|$. Therefore, for the rest of this subsection, we assume that $\left|V_{T}\right| \leq\left|V_{O}\right|$. Our main result states that even the problem of determining existence of a valid task assignment is difficult (in particular, NPcomplete.) In order to prove this, we consider a subproblem in which all instances have $\left|V_{T}\right|=\left|V_{O}\right|$, and we will prove that this subproblem is NP-complete. Thus, for the rest of this subsection, we further assume that $\left|V_{T}\right|=\left|V_{O}\right|$. That is, the mapping $i \mapsto \sigma_{i}$ is a permutation.

Theorem 4.1: The problem of deciding whether there exists a mapping $\sigma$ such that the organizational structure $\left(G_{O}, \sigma\right)$ is valid with respect to a given task graph $G_{T}$ is NP-complete.

Proof: That the problem belongs to NP is evident: if we have a YES instance, the mapping $\sigma$ provides a certificate.

We now note that the problem of interest is equivalent to the following:

Problem P: Does there exist a permutation $i \mapsto \sigma_{i}$ such that whenever $(i, j) \in A_{T}$, then the distance of $\sigma_{i}$ and $\sigma_{j}$ (in the graph $G_{O}$ ) is at most 2.

For any graph $G$, let $T(G)$ be a graph with the same set of nodes and such that $(i, j)$ is an arc of $T(G)$ if and only if the distance of $i$ and $j$ in the graph $G$ is at most two. We then see that we are dealing with the following problem: 
Problem P': Given two graphs $G_{T}$ and $G_{O}$ with the same number of nodes, is $G_{T}$ isomorphic with a subgraph of $T\left(G_{O}\right)$ ?

We recall the problem, CLIQUE: Given a graph $G=(V, A)$, and a positive integer $k \leq|V|$, does $G$ have a clique of size $k$ ? CLIQUE is known to be NP-complete [GareyJ79]. We now consider a subproblem of CLIQUE, which we will call "Restricted Clique."

Restricted Clique: Given a graph $G^{\prime}=\left(V^{\prime}, A^{\prime}\right)$ in which the degree of each node is at least $\left|V^{\prime}\right| / 2+1$, and an integer $k^{\prime}$ such that $\left|V^{\prime}\right| / 2+2 \leq k^{\prime} \leq\left|V^{\prime}\right|$, does $G^{\prime}$ have a clique of size $k^{\prime}$ ?

Lemma 1: "Restricted Clique" is NP-complete.

Proof: Let there be given an instance $(G, k)$ of the CLIQUE problem and let $m$ be the number of nodes of $G$. We construct a new graph $G^{\prime}$, as follows. The graph $G^{\prime}$ consists of the graph $G$ together with $m+4$ additional nodes. Each of these additional nodes are connected by means of an arc to every other node in $G^{\prime}$. Note that $G^{\prime}$ has $2 m+4$ nodes - i.e., $\left|V^{\prime}\right|=2 m+4$. Also, note that the degree of each node in $G^{\prime}$ is at least $m+3=\left|V^{\prime}\right| / 2+1$. Construct $k^{\prime}$ by $k^{\prime}=m+4+k$. Note that $k^{\prime} \geq m+4=\left|V^{\prime}\right| / 2+2$ and $k^{\prime}=m+4+k \leq m+4+|V|=\left|V^{\prime}\right|$. These constructions can be completed in polynomial time. If $G$ has a clique of size $k$, then the nodes in this clique and the $m+4$ added nodes form a clique of size $k+m+4=k^{\prime}$ in $G^{\prime}$. For converse, suppose $G^{\prime}$ has a clique of size $k^{\prime}=k+m+4$. Then, among the $k^{\prime}$ nodes in this clique, the number of nodes added in constructing $G^{\prime}$ can be at most $m+4$. Therefore, at least $k^{\prime}-(m+4)=k$ nodes are in $G$ and fully connected. Thus, it is implied that $G$ has a clique of size $k$. We have thus reduced the general CLIQUE problem to the "Restricted Clique" problem. Then, because CLIQIUE is in NPcomplete, "Restricted Clique" is in NP-complete. Q.E.D.

Recall now the SUBGRAPH ISOMORPHISM problem: given two graphs $G$ and $G^{\prime}$, is $G$ isomorphic to a subgraph of $G^{\prime}$ ? Now we consider a subproblem of SUBGRAPH 
ISOMORPHISM. Since CLIQUE is a special case of SUBGRAPH ISOMORPHISM, which we call "Restricted Subgraph Isomorphism."

Restricted Subgraph Isomorphism: Let $G=(V, A)$ and $G^{\prime}=\left(V^{\prime}, A^{\prime}\right)$ be graphs in which the degree of each node is at least $\left|V^{\prime}\right| / 2+1$ and such that $|V| \leq\left|V^{\prime}\right|$. Is $G$ isomorphic to a subgraph of $G^{\prime}$ ?

Lemma 2: "Restricted Subgraph Isomorphism" is NP-complete.

Proof: We can further restrict the instances of this problem so that $G$ must be a fully connected graph with $k^{\prime}$ nodes, where $k^{\prime}$ is some integer such that $\left|V^{\prime}\right| / 2+2 \leq k^{\prime} \leq\left|V^{\prime}\right|$. Then, the degree of each node in $G$ is $k^{\prime}-1 \geq\left|V^{\prime}\right| / 2+1$. This subproblem of the "Restricted Subgraph Isomorphism" is the "Restricted Clique" problem, which is NP-complete. Therefore, the "Restricted Subgraph Isomorphism" is NP-complete. Q.E.D.

We will need another graph transformation. Given a graph $G$, we denote by $Q(G)$ the graph which is the same as $G$ except that each arc of $G$ is replaced by a sequence of $3 \operatorname{arcs}$, as shown in Fig. 2. We introduce some more notation. If $G=(V, A)$ is a graph and $i \in V$ is a node of that graph, we use $T(Q(i))$ to denote the image of node $i$ when the transformations $Q$ and $T$ are applied in succession. Some nodes in graph $T(Q(G))$ is of the from $T(Q(i))$ with $i \in V$, and other nodes in $T(Q(G))$ are not in that form. 


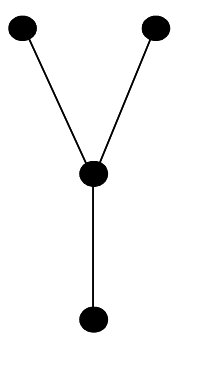

a)

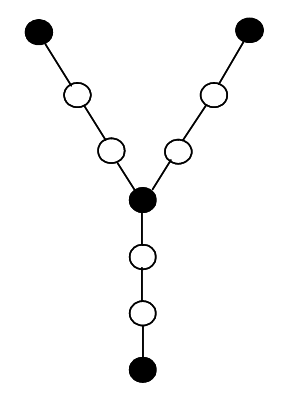

b)

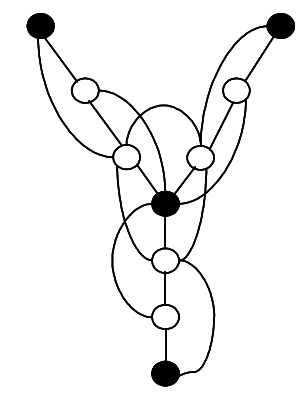

c)

Fig. 2. a) A graph $G ; \quad$ b) the graph $Q(G) ; \quad$ c) the graph $T(Q(G))$.

Lemma 3: Let $G=(V, A)$ be a graph in which all nodes have degree at least $d$.

(a) If $i$ is a node of $G$, then node $T(Q(i))$ has degree at least $2 d$; all nodes of $T(Q(G))$ that are not of the form $T(Q(i))$ for some $i \in V$ have degree bounded above by $|V|+1$.

(b) If $(i, j)$ is an arc of $G$, then the distance in the graph $T(Q(G))$ between $T(Q(i))$ and $T(Q(j))$ is equal to 2 ; if $(i, j)$ is not an arc of $G$, then the distance between $T(Q(i))$ and $T(Q(j))$ is larger than 2 . 
Proof: a) If a node in $G$ has degree $\delta \geq d$, then the corresponding node in $T(Q(G))$ is connected to its neighbors in $Q(G)$ (there are $\delta$ of them) and to the neighbors of these neighbors (there are $\delta$ of them as well, for a total of $2 \delta>2 d$.

Note that $Q(G)$ and $T(Q(G))$ have an identical set of nodes. If a node in $T(Q(G))$ is not of the form $T(Q(i))$, then it has only 2 neighbors in the graph $Q(G)$. One of these neighbors has a single extra neighbor; the other one corresponds to a node of the original graph $G$ and has at most $|V|-2$ extra neighbors in $Q(G)$. Thus, the degree of the node under consideration in $T(Q(G))$ is at most $2+1+(|V|-2)=|V|+1$.

b) Evident from Fig. 2. Q.E.D.

Consider a graph $G=(V, A)$ in which each node has degree at least $|V| / 2+1$. Then, in the graph $T(Q(G))$, nodes of the form $T(Q(i))$ will have degree at least $|V|+2$. All other nodes in $T(Q(G))$ will have degree at most $|V|+1$. Thus, for each node of $T(Q(G))$, it can be immediately determined whether it is of the form $T(Q(i))$ or not.

Lemma 4: Let $G=(V, A)$ and $G^{\prime}=\left(V^{\prime}, A^{\prime}\right)$ be graphs in which the degree of each node is at least $\left|V^{\prime}\right| / 2+1$ and such that $|V| \leq\left|V^{\prime}\right|$. Then, $G$ is isomorphic to a subgraph of $G^{\prime}$ if and only if $T(Q(G))$ is isomorphic to a subgraph of $T\left(Q\left(G^{\prime}\right)\right)$.

Proof: If $G$ is isomorphic to a subgraph of $G^{\prime}$, it is evident that $T(Q(G))$ is isomorphic to a subgraph of $T\left(Q\left(G^{\prime}\right)\right)$. It only remains to prove the reverse implication.

Suppose that $T(Q(G))$ is isomorphic to a subgraph of $T\left(Q\left(G^{\prime}\right)\right)$. Consider any node of $T(Q(G))$ which has degree larger than $\left|V^{\prime}\right|+1$. Then, the degree of this node is larger than $|V|+1$ because $|V| \leq\left|V^{\prime}\right|$. Such a node is of the form $T(Q(i))$ for some node $i$ of $G$, by Lemma 3(a). Since $T(Q(G))$ is isomorphic to a subgraph of $T\left(Q\left(G^{\prime}\right)\right)$, node $T(Q(i))$ is mapped to 
some node of $T\left(Q\left(G^{\prime}\right)\right)$ that has also degree larger than $\left|V^{\prime}\right|+1$, and is therefore of the form $T\left(Q\left(i^{\prime}\right)\right)$, where $i^{\prime}$ is a node of $G^{\prime}$.

Suppose that $(i, j)$ is an arc of $G$. Then, because nodes $i$ and $j$ in graph $G$ have degree at least $\left|V^{\prime}\right| / 2+1, T(Q(i))$ and $T(Q(j))$ have both degree larger than $2\left(\left|V^{\prime}\right| / 2+1\right)-1=\left|V^{\prime}\right|+1$ (by Lemma 3(a)). Also, their distance in $T(Q(G))$ is equal to 2 (by Lemma 3(b).) Since $T(Q(G))$ is a subgraph of $T\left(Q\left(G^{\prime}\right)\right)$, the nodes $T(Q(i))$ and $T(Q(j))$ are mapped to some (distinct) nodes in $T\left(Q\left(G^{\prime}\right)\right)$ which are of degree larger than $\left|V^{\prime}\right|+1$. In particular, these latter nodes of $T\left(Q\left(G^{\prime}\right)\right)$ must be of the form $T\left(Q\left(i^{\prime}\right)\right)$ and $T\left(Q\left(j^{\prime}\right)\right)$, for some nodes $i^{\prime}$ and $j^{\prime}$ of $G^{\prime}$. Since the distance between $T(Q(i))$ and $T(Q(j))$ is equal to 2 , the distance of $T\left(Q\left(i^{\prime}\right)\right)$ and $T\left(Q\left(j^{\prime}\right)\right)$ must be at most 2. Using Lemma 3(b), we conclude that $\left(i^{\prime}, j^{\prime}\right)$ is an arc of $G^{\prime}$. Therefore, by mapping $i$ and $j$ to $i^{\prime}$ and $j^{\prime}$, respectively, and by mapping similarly all other nodes of $G$ to nodes of $G^{\prime}$, we see that $G$ is isomorphic to a subgraph of $G^{\prime}$, which concludes the proof of the lemma. Q.E.D.

We notice that Lemma 4 facilitates polynomially transforming the "Restricted Subgraph Isomorphism" problem (shown earlier to be NP-complete) to Problem $\boldsymbol{P}^{\prime}$. We now consider this polynomial transformation. For an instance of the "Restricted Subgraph Isomorphism" problem such that $|V|=\left|V^{\prime}\right|$, construct the instance of Problem $\boldsymbol{P}^{\prime}, G_{0}$ and $G_{T}$, by $G_{0}=Q\left(G^{\prime}\right)$ and $G_{T}$ $=T(Q(G))$. By Lemma 4, $G$ is isomorphic with a subgraph of $G^{\prime}$ if and only if $G_{T}$ is isomorphic with a subgraph of $T\left(G_{O}\right)$. For an instance of the "Restricted Subgraph Isomorphism" problem such that $|V|<\left|V^{\prime}\right|$, construct $G_{0}=Q\left(G^{\prime}\right)$ and construct $G_{T}$ by adding zero-degree nodes to $T(Q(G))$ so that $G_{0}$ and $G_{T}$ have the same number of nodes. Then, $G_{T}$ is isomorphic with a subgraph of $T\left(G_{O}\right)$ if and only if $T(Q(G))$ is isomorphic with a subgraph of $T\left(Q\left(G^{\prime}\right)\right)$. Then, by Lemma 4, $T(Q(G))$ is isomorphic with a subgraph of $T\left(Q\left(G^{\prime}\right)\right)$ if and only if $G$ is isomorphic with a subgraph of $G^{\prime}$. Because of this polynomial transformation, we can conclude that Problem $\boldsymbol{P}^{\prime}$ is NP-complete, and the proof of Theorem 4.1 has been completed. Q.E.D. 
We have shown that it is difficult to even determine whether a valid organizational structure, as defined in section 1, does exist. It follows that the problem of determining an optimal valid organization is also difficult (NP-hard), for any nontrivial choice of the performance criterion.

\section{THE CASE WHERE ONLY THE NUMBER OF NODES IN $G_{O}$ IS FIXED}

We now consider the case where $G_{T}$ is given and the system designer has the flexibility of designing the network topology of the agents, $G_{O}=\left(V_{O}, A_{O}\right)$. This problem models a situation in which a government needs to design an organizational structure to perform a huge global task. We assume that the number of agents, $\left|V_{o}\right|$, is given, and the problem is to find graph $G_{O}=\left(V_{O}, A_{O}\right)$ and mapping $\sigma: V_{T} \cup A_{T} \rightarrow V_{O}$. As in sections 3 and 4 , in this section we also assume that each agent can have at most one subtask. Therefore, if $\left|V_{T}\right|>\left|V_{O}\right|$, there is obviously no feasible mapping $\sigma$. For the rest of this section, we assume that $\left|V_{T}\right| \leq\left|V_{O}\right|$. No other constraints are imposed on $G_{O}-$ i.e., $A_{O}$ is not given.

Under the above constraints, the problem of designing a valid organizational structure that minimizes $C 1$ is trivial. Let us denote $V_{T}=\{1,2, \ldots, m\}, V_{O}=\left\{a_{1}, a_{2}, \cdots, a_{n}\right\}$. We can pick $m$ nodes from $V_{O}$ and construct an organizational graph,

$$
G_{O}=\left(\left\{a_{1}, a_{2}, \cdots, a_{m}\right\},\left\{\left(a_{1}, a_{2}\right),\left(a_{1}, a_{3}\right),\left(a_{1}, a_{4}\right), \cdots,\left(a_{1}, a_{m}\right)\right\}\right) \text { (a "star" graph), }
$$

and let $\sigma_{i j}=a_{1}$ for all $(i, j) \in A_{T}$. We then have $C 1=m-1$. Since $\mathrm{G}_{T}$ is connected, as mentioned in section 1, it is clear that $G_{O}$ must also be connected and therefore no valid organization could have less than $m-1$ arcs. Therefore, the minimum value of $C 1$ is $m-1$.

If we impose a load balancing constraint $L \leq L_{u p}$ and attempt to minimize $C l$ subject to that constraint, we obtain an apparently more difficult problem. We conjecture that this problem is NP-hard [GareyJ79], although we have not been able to establish this result.

The following theorem addresses minimizing $C 2$. 
Theorem 5.1: Under the assumptions of this section, the problem of designing a valid organizational structure in which $C 2$ is minimized subject to the constraint $L \leq L_{u p}$, can be formulated as a min-cost linear network flow problem and can be solved in polynomial time.

Proof: Because $G_{O}$ is not given as a constraint, the task assignment process can pick $G_{O}$ to be a complete (fully connected) graph in order to minimize $C 2$. With the fully connected $G_{O}$, we can minimize $C 2$ subject to the constraint $L \leq L_{u p}$. However, this problem is a special case of the problem considered in section 3.2.1, and the result follows from section 3.2.1 Q.E.D.

\section{ALLOWING MULTIPLE SUBTASKS FOR AN AGENT}

So far in this paper, we have discussed the task assignment problem under the assumption that an agent cannot have more than one subtask. We now briefly discuss the problems with that assumption relaxed.

\subsection{Fixed Organization}

As in section 3.1, we now assume that the organizational graph $G_{O}$ is fixed and that the assignment of subtasks, $\sigma_{i}, \forall i \in V_{T}$, is predetermined. The difference of these assumptions from those in section 3.1 is that the task assigner may face the case that $\sigma_{i}=\sigma_{j}$ even if $i \neq j$. Therefore, the task assignment problem for a fixed organization under discussion now is a generalization of the problem addressed in section 3.1. The generalized problem can be solved by simply adjusting Algorithm 3.1 as follows:

\section{Algorithm 6.1 - Phase 1}

- For each element $(i, j)$ of $A_{T}$, create a node $m_{i j}$, and for each element $a_{i}$ of $V_{O}$, create a node $d_{i}$.

- For each element $(i, j)$ of $A_{T}$ and for each node $a_{k} \in V_{O}$, if $\left(\sigma_{i}, \sigma_{k}\right) \in A_{O}$ and $\left(\sigma_{j}, \sigma_{k}\right) \in A_{O}$, then create an edge from $m_{i j}$ to $d_{k}$.

- Create the source node $s$ and make an edge from $s$ to each $m_{i j}$ with capacity limit 1.

- Create the sink node $t$

- For each agent $a_{k}$, make an edge from node $d_{k}$ to $t$ with capacity $\operatorname{limit} L_{u p}-\sum_{\left\{i \in V_{T} \mid \sigma_{i}=a_{k}\right\}} p(i)$. 
Phase 2 solves the max-flow problem associated with this flow graph.

\section{Algorithm 6.1 - Phase 2}

- Run an algorithm that solves, under the constraint of integer flows, the max-flow problem constructed in Phase 1.

- If the maximal flow is $\left|A_{T}\right|$, there is a valid task assignment. Otherwise, there is not.

\subsection{Fixed Organization of Versatile Agents}

In this subsection, we consider the task assignment problem in the organization of versatile agents without any constraint on which agent can be assigned which tasks. We make no assumption about the relative sizes of $\left|V_{T}\right|$ and $\left|V_{O}\right|$. In this problem, a valid organizational structure, per Definition 1, obviously exists. (For example, if all subtasks in $V_{T}$ and all interactions in $A_{T}$ are assigned to a single agent, the resulting organizational structure is valid.)

\subsubsection{Minimizing the maximum load $L$}

We formulate the task assignment problem in the fixed organization of versatile agents as an integer linear programming problem. For each subtask $i \in V_{T}$ and each agent $a_{k} \in V_{O}$, we define one binary integer variable $x_{i, k} \in\{0,1\}$. The expression $x_{i, k}=1$ signifies that $\sigma_{i}=a_{k}$. For each $(i, j) \in A_{T}$ and each agent $a_{k} \in V_{O}$, we define one binary integer variable $x_{i j, k} \in\{0,1\}$. The expression $x_{i j, k}=1$ signifies $\sigma_{i j}=a_{k}$, and $x_{i j, k}=0$ signifies that the interaction between subtasks $i$ and $j$ is not handled by agent $a_{k}$. We formulate the following ILP constraints:

$\sum_{k} x_{i, k}=1, \forall i \in V_{T}$

$\sum_{k} x_{i j, k}=1, \forall(i, j) \in A_{T}$

Note that if $\sigma_{i j}=a_{k}$, then both $\left(a_{k}, \sigma_{i}\right)$ and $\left(a_{k}, \sigma_{j}\right)$ must be in $A_{O}$ in order for mapping (task assignment) $\sigma$ to be feasible. This constraint in terms of ILP variables can be expressed as follows:

$$
\begin{array}{r}
x_{i j, k} \leq \sum_{a_{l} \in H\left(a_{k}\right)} x_{i, l} \text { and } x_{i j, k} \leq \sum_{a_{l} \in H\left(a_{k}\right)} x_{j, l}, \\
\forall(i, j) \in A_{T}, \forall a_{k} \in V_{O},
\end{array}
$$


where we denote the set of all neighboring nodes of agent $a_{k} \in V_{O}$ as

$$
H\left(a_{k}\right)=\left\{a_{l} \in V_{O} \mid\left(a_{l}, a_{k}\right) \in A_{O}\right\} .
$$

In summary, the ILP problem is:

minimize $L$

subject to

$$
\begin{aligned}
& L \geq \sum_{i \in V_{T}} x_{i, k} p(i)+\sum_{(i, j) \in A_{T}} x_{i j, k}, \quad \forall a_{k} \in V_{O} \\
& \sum_{k} x_{i, k}=1, \forall i \in V_{T} \\
& \sum_{k} x_{i j, k}=1, \forall(i, j) \in A_{T} \\
& x_{i j, k} \leq \sum_{a_{l} \in H\left(a_{k}\right)} x_{i, l} \text { and } x_{i j, k} \leq \sum_{a_{l} \in H\left(a_{k}\right)} x_{j, l}, \\
& \qquad(i, j) \in A_{T}, \forall a_{k} \in V_{O} \\
& x_{i j, k} \in\{0,1\}, \forall(i, j) \in A_{T}, \forall a_{k} \in V_{O} \\
& x_{i, k} \in\{0,1\}, \forall i \in V_{T}, \forall a_{k} \in V_{O}
\end{aligned}
$$

We can use available algorithms to solve this integer linear programming problem. However, whether there is a polynomial-time algorithm for this particular ILP problem is unknown.

\subsubsection{Minimizing a communication measure}

The problem of minimizing the number $C 1$ of arcs is vacuous because $G_{\mathrm{O}}$ is assumed to be given and therefore $C 1$ is predetermined. The problem of minimizing the number $C 2$ without a further constraint is also trivial. If we choose a constant mapping $\sigma_{i}=\sigma_{i j}=a_{1}, \forall i \in V_{T}, \forall(i, j) \in A_{T}$,

(that is, to assign all subtasks and all interactions to one agent), then $C 2=0$. However, this mapping places all of the load on a single agent. Thus, we now consider minimizing $C 2$ subject to an upper bound $L_{u p}$ on the maximum load $L$. We can formulate this problem as an integer programming problem similar to the one formulated in section 6.2.1:

$\operatorname{minimize} \sum_{(i, j) \in A_{T}} \sum_{a_{l} \in V_{O}} \sum_{a_{m} \in V_{O}} \sum_{a_{k} \in V_{O}} \mu\left((i, j), a_{l}, a_{m}, a_{k}\right) x_{i, l} x_{j, m} x_{i j, k}$

subject to 


$$
\begin{aligned}
& L_{u p} \geq \sum_{i \in V_{T}} x_{i, k} p(i)+\sum_{(i, j) \in A_{T}} x_{i j, k}, \quad \forall a_{k} \in V_{O} \\
& \sum_{k} x_{i, k}=1, \forall i \in V_{T} \\
& \sum_{k} x_{i j, k}=1, \forall(i, j) \in A_{T} \\
& x_{i j, k} \leq \sum_{a_{l} \in H\left(a_{k}\right)} x_{i, l} \text { and } x_{i j, k} \leq \sum_{a_{l} \in H\left(a_{k}\right)} x_{j, l}, \\
& \qquad \forall(i, j) \in A_{T}, \forall a_{k} \in V_{O} \\
& x_{i j, k} \in\{0,1\}, \forall(i, j) \in A_{T}, \forall a_{k} \in V_{O} \\
& x_{i, k} \in\{0,1\}, \forall i \in V_{T}, \forall a_{k} \in V_{O}
\end{aligned}
$$

The main difference between this problem and the ILP problem in section 6.2.1 is that the objective function in this problem is nonlinear.

\subsection{The Case in Which Only the Number of Nodes in $G_{O}$ Is Fixed}

Now we consider the case in which the system designer determines the organizational graph $\boldsymbol{G}_{\boldsymbol{O}}$ as well as mapping $\sigma$. Because we have two objectives (load balancing and minimizing the communication measure), we can consider two types of optimization problem formulations:

1) Minimizing the communication measure ( $C 1$ or $C 2$ ) under constraint $L<L_{u p}$, where $L_{u p}$ is the maximum allowed load for an individual agent.

2) Minimizing $L$ under the constraint $C 1 \leq C 1_{u p}$ (or under the constraint $C 2 \leq C 2_{u p}$ )

In this section, we establish that a polynomial-time algorithm is not likely to exist for either of these two problems. In order to establish that, we first convert these problems into the following single decision [PaSt82] problem:

Problem 6.1: Given $G_{T}=\left(V_{T}, A_{T}\right)$, the number of agents $\left|V_{o}\right|, L_{u p}$, and $C 1_{u p}\left(\right.$ or $\left.C 2_{u p}\right)$, is there a valid organizational structure that results in $L<L_{u p}$ and $C 1 \leq C 1_{u p}$ (or $\left.C 2 \leq C 2_{u p}\right)$ ?

We will prove that this problem is NP-complete. To do so, we consider a subproblem in which $C 1_{u p}$ (or $C 2_{u p}$ ) is sufficiently large that the constraint on the communication measure is never violated for any choice of valid organizational structure, e.g.,

$C 1_{u p} \geq\left|V_{o}\right|\left(\left|V_{o}\right|-1\right) / 2$,

$C 2_{u p} \geq\left|A_{T}\right| \max _{(i, j) \in A_{T}, a_{l}, a_{m}, a_{k}} \mu\left((i, j), a_{l}, a_{m}, a_{k}\right)$. 
In such a subproblem, the communication constraint plays no role. The following theorem proves that this subproblem is NP-complete and thus that Problem 6.1 is also NP-complete.

Theorem 6.1 The problem of deciding whether there exists a mapping $\sigma$ such that

$$
\ell_{k} \equiv \sum_{\left\{i \in V_{T} \mid \sigma_{i}=k\right\}} p(i)+\left|\left\{(i, j) \in A_{T} \mid \sigma_{i j}=a_{k}\right\}\right| \leq L_{u p}, \forall a_{k} \in V_{O}
$$

for a given task graph $G_{T}$, set of agents $V_{O}$, and $L_{u p}$, is NP-complete.

Proof: We recall an NP-complete problem, Bin Packing [GareyJ79].

Bin Packing: Given a set $U=\left\{u_{1}, u_{2}, \cdots, u_{m}\right\}$ of items, a positive integer size $s(u)$ for each $u \in U$, a positive integer bin capacity $B$, and a positive integer $K$, is there a partition of $U$ into disjoint sets $U_{1}, U_{2}, \ldots, U_{K}$ such that the sum of the sizes of the items in each $U_{i}$ is $B$ or less?

Now we describe a polynomial transformation from the Bin Packing problem to our task assignment problem under discussion. Construct the set of subtasks $V_{T}=U$. For each subtask $u \in V_{T}$, define $p(u)=\alpha s(u)-m$, where $\alpha$ is an integer such that $\alpha>>m^{2}$. Construct $A_{T}=\left\{\left(u_{1}, u_{2}\right),\left(u_{2}, u_{3}\right), \cdots,\left(u_{m-1}, u_{m}\right)\right\}$ so that $G_{T}=\left(V_{T}, A_{T}\right)$ is connected. (Thus, $\left.\left|A_{T}\right|=m-1.\right)$ Construct a set, $V_{O}=\left\{a_{1}, a_{2}, \cdots, a_{K}\right\}$, of $K$ agents. (Note that agent $a_{k}$ corresponds to set $U_{k}$.) Let $L_{u p}=\alpha B$. These constructions can be completed in polynomial time.

Suppose that $U$ can be partitioned into disjoint sets $U_{1}, U_{2}, \ldots, U_{K}$ such that the sum of the sizes of the items in each $U_{k}$ is $B$ or less. Then we have $\sum_{u \in U_{k}} s(u) \leq B$ for each $k=1,2, \cdots, K$. Consider a mapping $\sigma: U \cup A_{T} \rightarrow V_{O}$ (or equivalently, $\sigma: V_{T} \cup A_{T} \rightarrow V_{O}$ ) such that $\sigma_{u}=a_{k}$ if item $u$ is in $U_{k}$ in the Bin Packing instance, and such that $\sigma_{i j}=a_{*}, \forall(i, j) \in A_{T}$ for some agent $a_{*}$ that is assigned with at least one subtask. Now, for agent $a_{*}$,

$$
\begin{aligned}
\ell_{*} & =\left|A_{T}\right|+\sum_{\left\{u \in V_{T} \mid \sigma_{u}=a_{*}\right\}} p(u) \\
& =(m-1)+\sum_{\left\{u \in U_{*}\right\}}[\alpha s(u)-m] \\
& \leq \alpha \sum_{\left\{u \in U_{*}\right\}} s(u) \leq \alpha B=L_{u p}
\end{aligned}
$$

For an arbitrary agent $a_{k} \neq a_{*}$, 


$$
\begin{aligned}
\ell_{k} & =\sum_{\left\{u \in V_{T} \mid \sigma_{u}=a_{k}\right\}} p(u) \\
& =\sum_{\left\{u \in U_{k}\right\}}[\alpha s(u)-m] \\
& = \begin{cases}-m\left|U_{k}\right|+\alpha \sum_{\left\{u \in U_{k}\right\}} s(u) & \text { if }\left|U_{k}\right|>0 \\
0 & \text { if }\left|U_{k}\right|=0\end{cases} \\
& \leq \alpha B=L_{u p}
\end{aligned}
$$

Therefore, there exists a mapping $\sigma$ that results in the maximal load less than or equal to $L_{u p}$.

Suppose there exists no partition of $U$ into disjoint sets $U_{1}, U_{2}, \ldots, U_{K}$ such that the sum of the sizes of the items in each $U_{k}$ is $B$ or less. Then, in every way $U$ is partitioned, $\sum_{u \in U_{k}} s(u) \geq B+1$ for some $U_{k}$. Consider an arbitrary mapping $\sigma$. Then $U_{k} \equiv\left\{u \in V_{T} \mid \sigma_{u}=a_{k}\right\}$, $k=1,2, \ldots, K$ is a partition of $U$; namely, in this corresponding partition, each item $u$ in $U=V_{T}$ is in $U_{k}$ if $\sigma_{u}=a_{k}$. For some $U_{k}, \sum_{u \in U_{k}} s(u) \geq B+1$, which is equivalent to $\sum_{u \in U_{k}} \alpha s(u) \geq \alpha B+\alpha$. Therefore, for some $a_{k}$,

$$
\begin{aligned}
& \sum_{\left\{u \in V_{T} \mid \sigma_{u}=k\right\}} p(u) \\
= & \sum_{\left\{u \in V_{T} \mid \sigma_{u}=a_{k}\right\}}[\alpha s(u)-m] \\
\geq & \sum_{\left\{u \in V_{T} \mid \sigma_{u}=a_{k}\right\}}[\alpha s(u)]-m^{2} \\
\geq & \alpha(B+1)-m^{2} \\
= & \alpha B+\alpha-m^{2}>\alpha B=L_{u p}
\end{aligned}
$$

Therefore, for some agent $a_{k}$, we have

$$
\begin{aligned}
\ell_{k} & \equiv \sum_{\left\{u \in V_{T} \mid \sigma_{u}=a_{k}\right\}} p(u)+\left|\left\{(i, j) \in A_{T} \mid \sigma_{i j}=a_{k}\right\}\right| \\
& >L_{u p}
\end{aligned}
$$

This implies that for every mapping $\sigma$, the maximum load is strictly more than $L_{u p}$. Q.E.D.

We have considered a subproblem in which $C 1_{u p}\left(\right.$ or $\left.C 2_{u p}\right)$ is sufficiently large and proved that this subproblem is NP-complete. Now we briefly discuss other specialized subproblems of Problem 6.1. Without a constraint on the load, minimizing $C 1$ or $C 2$ is a trivial problem. If we assign all subtasks and interactions to a single agent, we have $C 1=C 2=0$. If we impose a load- 
balancing constraint $L \leq L_{u p}$, where $L_{u p}<\sum_{i \in V_{T}} p(i)+\left|A_{T}\right|$, and attempt to minimize $C$, then we obtain an apparently more difficult problem, but we may have an efficient algorithm to solve it. We leave this problem for future study. Also, we consider minimizing $C 2$ under the same loadbalancing constraint $L \leq L_{u p}$. In this case, we can use the fully connected $G_{O}$ for the purpose of minimization because there is no constraint on the physical connectivity of $G_{O}$. In contrast to Theorem 5.1, this problem is apparently difficult. This problem is again left for future study.

\section{CONCLUSIONS}

This paper formulated a new class of design problems for distributed systems. We have derived solution procedures for some of these design problems, and we have seen that another variation leads to NP-hard problems. It is believed that these formulations capture some generic features of distributed system design problems.

\section{ACKNOWLEDGMENT}

The author would like to thank Prof. John Tsitsiklis at the Massachusetts Institute of Technology for his valuable advisement.

\section{REFERENCES}

[AlPrRa99] A.H. Alhusaini, Viktor K. Prasanna, and C.S. Raghavendra, "A unified resource scheduling framework for heterogeneous computing environments," 8th Heterogeneous Computing Workshop (HCW'99), Apr. 1999.

[AngerHC90] F. D. Anger, J. J. Hwang, and Y. C. Chow, "Scheduling with sufficiently loosely coupled processors," J. Parallel and Distributed Computing, vol. 9, no. 1, 1990, pp. 87-92.

[ArmHK98] R. Armstrong, D. Hensgen, and T. Kidd, "The relative performance of various mapping algorithm is independent of sizable variance in run-time predictions," 7th Heterogeneous Computing Workshop (HCW'98), March 1998.

[BaJS05] M. S. Bazaraa, J. J. Jarvis, and H. D. Sherali, Linear Programming and Network Flows, Wiley, Hoboken, NJ, 2005.

[BatA194] S. Bataineh and B. Al-Alsir, "An efficient scheduling algorithm for divisible and indivisible tasks in loosely coupled multiprocessor systems," Software Engineering J., vol. 9, 1994, pp. 13-18.

[Ber98] D. P. Bertsekas, Network Optimization: Continuous and Discrete Models, Athena Scientific, Belmont, MA, 1998. 
[BerGa192] D. Bertsekas and R. Gallager, Data Networks, $2^{\text {nd }}$ Ed., Prentice Hall, Englewood Cliffs, NJ, 1992.

[BerTsi89] D. P. Bertsekas and J. N. Tsitsiklis, Parallel and distributed computation: numerical methods, Prentice Hall, Englewood Cliffs, N.J, 1989.

[BerTsi97] D. Bertsimas and J. N. Tsitsiklis, Introduction to Linear Optimization, Athena Scientific, Belmont, MA, 1997.

[BerW96] F. Berman and R. Wolski, "Scheduling from the perspective of the application," $5^{\text {th }}$ IEEE International Symposium on High Performance Distributed Computing, Aug. 1996.

[BhGM95] V. Bharadwaj, D. Ghose, and V. Mani, "An efficient load distribution strategy for a distributed linear network of processors with communication delays," Computers and Mathematics with Applications, vol. 29, no. 9, 1995, pp. 95-112.

[B1Dr96] J. Blazewicz and M. Drozdowski, "The performance limits of a two-dimensional network of load sharing processors," Foundations of Computing and Decision Sciences, vol. 21, no.1, 1996, pp. $3-15$.

[Bok81] S. Bokhari, "A shortest tree algorithm for optimal assignments across space and time in a distributed processor system," IEEE Transactions on Software Engineering, vol. 7, no. 6. pp. 583-589, 1981.

[Bok87] Bokhari, S.H., Assignment Problems in Parallel and Distributed Computing, Kluwer Academic Publishers, Boston, MA, 1987.

[Braun98] T. D. Braun et al., "A taxonomy for describing matching and scheduling heuristics for mixedmachine heterogeneous computing systems," IEEE Workshop on Advances in Parallel and Distributed Systems, West Lafayette, IN. Oct. 1998. pp. 330-335.

[CasKu88] T. L. Casavant and J. G. Kuhl, "A taxonomy of scheduling heuristics in general-purpose distributed computing systems," IEEE Trans. Software Engineering, vol. 14, no. 2, Feb. 1988.

[ChuHLE80] W. W. Chu, L. J. Holloway, M. Lan, and K. Efe, "Task allocation in distributed data processing," IEEE Computer, pp. 57-69, Nov. 1980.

[Diek93] R. Diekmann, R. Lüling, A. Reinefeld, "Distributed combinatorial optimization," Proc. SOFSEM'93, Hrdoňov Šumava, Czech Republic, 1993, pp. 33-60.

[Efe82] K. Efe, "Heuristic models of task assignment scheduling in distributed systems," IEEE Computer, June 1982, pp.50-56.

[Fer89] D. Fernández-Baca, "Allocating modules to processors in a distributed system," IEEE Transactions on Software Engineering, vol. 15, no. 11, Nov. 1989, pp. 1427-1436. 
[Fre96] R. Freund, B. Carter, D. Watson, E. Keith, and F. Mirabile, "Generational scheduling for heterogeneous computing systems," International Conf. Parallel and Distributed Processing Techniques and Applications (PDPTA'96), pp. 769-778, Aug. 1996.

[GareyJ79] M. R. Garey and D. S. Johnson, Computers and Intractability, W. H. Freeman and Company, New York, 1979.

[Grid99] I. Foster and C. Kesselman, ed., The Grid: blueprint for new computing infrastructure, Morgan Kaufmann Publishers, San Francisco, CA, 1999.

[Gus83] D. Gusfield, "Parametric combinatorial computing and a problem in module distribution," Journal of the Association for Computing Machineray, vol. 30, no. 3, July, 1983, pp. 551-563.

[IvOzFo95] M. Iverson, F. Ozguner, and G. J. Follen, "Parallelizing existing applications in a distributed homogeneous environment," 4th Heterogeneous Computing Workshop (HCW '95), pp. 93-100, April 1995.

[LePS95] C. Leangsuksun, J. Potter, and S. Scott, "Dynamic task mapping algorithms for a distributed homogeneous computing environment," 4th Heterogeneous Computing Workshop (HCW'95), pp. 30-34, April 1995.

[Lee87] D. C. Lee, Task Allocation for Efficient Performance of a Decentralized Organization, Master's thesis, Massachusetts Institute of Technology, 1987, Dept. of Electrical Engineering and Computer Science.

[Lo88] V. M. Lo, "Heuristic algorithms for task assignment in distributed systems," IEEE Transactions on Computers, vol. 37, no. 11, Nov. 1988, pp.1384-1397.

[MaSie98] M. Maheswaran and H. J. Siegel, "A dynamic matching and scheduling algorithm for heterogeneous computing systems," $7^{\text {th }}$ Heterogeneous Computing Workshop (HCW'98), pp. 57-69, March 1998.

[Mon95] B. Monien, R. Diekmann, R. Feldmann, R. Klasing, R. Lüling, K. Menzel, T. Römke, U.-P. Schroeder, "Efficient use of parallel \& distributed systems: from theory to practice," Lecture Notes in Computer Science, No. 1000, Springer-Verlag, 1995.

[PaSt82] Papadimitriou, C.H. and Steiglitz, K., Combinatorial Optimization: Algorithms and Complexity, Prentice Hall, Englewood Cliffs, New Jersey, 1982.

[PengS93] D.-T. Peng and K. G. Shin, "Optimal scheduling of cooperative tasks in a distributed system using an enumerative method," IEEE Transactions on Software Engineering, vol. 19, no. 3, Nov. 1993, pp. 253-267.

[Röm96] T. Römke, M. Röttger, U.-P. Schroeder, and J. Simon, “On efficient embedding of grids into grids in PARIX," Proc. $1^{\text {st }}$ Int. Conf. on Parallel Processing, Euro-PAR'95, LNCS 966, Aug. 1996, pp. $181-192$. 
[ShHK95] B. A. Shirazi, A. R. Hurson, and K. M. Kavi, eds., Scheduling and Load Balancing in Parallel and Distributed Systems, IEEE Computer Society Press, Los Alamitos, CA, 1995.

[ShWP90] B. Shirazi, M. Wang, and G. Pathak, "Analysis and evaluation of heuristic methods for static task scheduling," Journal of Parallel and Distributed Computing, 10:222-232, 1990.

[SihLee93] G. C. Sih and E. A. Lee, "A compile-time schedule heuristic for interconnection-constrained heterogeneous processor architectures," IEEE Trans. Parallel and Distributed Systems, vol. 4, no. 2, Feb. 1993.

[Sinclair87] J. B. Sinclair, "Efficient computation of optimal assignments for distributed tasks," Journal of Parallel and Distributed Computing, vol. 4, no. 4, Aug. 1987, pp. 342-362.

[SohnR95] J. Sohn and T. G. Robertazzi, "An optimum load sharing strategy for divisible jobs with timevarying processor speed," Proc. Eighth International Conf. Parallel and Distributed Computer Systems, Orlando, FL, 1995, pp. 27-32.

[Stone77] H. S. Stone, "Multiprocessor scheduling with the aid of network flow algorithms," IEEE Transactions on Software Engineering, vol. 3, Jan. 1977, pp. 85-94.

[Stone78] H. S. Stone, "Critical load factors in two-processor distributed systems," IEEE Transactions on Software Engineering, vol. 4, May, 1978, pp. 254-258.

[Towsley86] D. Towsley, "Allocating problems containing branches and loops within a multiple processor system," IEEE Transactions on Software Engineering, vol. 12, no. 10, 1986, pp. 1018-1024.

[Tsi84] J. N. Tsitsiklis, Problems in Decentralized Decision Making and Computation, Ph.D. thesis, Dept. of EECS, MIT, Cambridge, MA, 1984.

[Wang97] L. Wang, H. J. Siegel, V. P. Roychowdhury, and A. A. Maciejewski, "Task matching and scheduling in heterogeneous computing environments using a genetic-algorithm-based approach," $J$. Parallel and Distributed Computing, 47(1):8-22, Nov. 1997.

[Weber93] R. Weber, "On a conjecture about assigning jobs to processes of different speeds," IEEE Trans. Automatic Control, vol. 38, no. 1, 1993, pp. 166-170.

[YangGe94] T. Yang and A. Gerasoulis, "DSC: scheduling parallel tasks on an unbounded number of processors," IEEE Trans. Parallel and Distributed Systems, vol. 5, no. 9, Sep. 1994. 
Daniel C. Lee (S'91-M'92) received the Ph.D. (1992) and M.S. (1987) degrees from the Massachusetts Institute of Technology in Electrical Engineering \& Computer Science. He received a B.S. (1985) degree in Electrical Engineering with honors and a B.S. (1985) degree in Mathematics from the University of Maryland at College Park. He is currently an Associate Professor in the School of Engineering Science at Simon Fraser University. His main research interests include quality of service and resource allocation issues in networks and communication systems. Applications of his research include wireless communications and networking, sensor networks, optical networks, and internet multimedia. He was previously an Assistant Professor in the Electrical Engineering Department of the University of Southern California. From 1993 to 1998 , Dr. Lee devoted his research to the systems engineering of networks and communication systems at the U.S. Naval Research Laboratory (NRL) in Washington, DC. At the Center for Computational Science in NRL, Dr. Lee participated in the development of an object oriented protocol software framework, CASiNO. At the Naval Space Center in NRL, Dr. Lee developed a proxy agent for managing the ICEbox network, a U.S. government information-dissemination system. Dr. Lee's honors include the Alan Berman Research Publication Award from NRL in 1995, the Navy's Outstanding Performance Award at NRL in 1995, and the Frederick C. Hennie III Teaching Award from MIT in 1989. 